\title{
Optimization of the Kinematic Chain of the Thumb for a Hand Prosthesis Based on the Kapandji Opposition Test
} \author{
Antonio Pérez-González ${ }^{1[0000-0003-0210-7728]}$ and Immaculada Llop-Harillo ${ }_{1947]}^{1[0000-0002-9378-}$ \\ ${ }^{1}$ Grupo de Biomecánica y Ergonomía, Departamento de Ingeniería Mecánica y Construcción, \\ Universitat Jaume I, 12071 Castellón, Spain \\ aperez@uji.es
}

\begin{abstract}
The thumb plays a key role in the performance of the hand for grasping and manipulating objects. In artificial hands the complex thumb's kinematic chain (TKC) is simplified and its five degrees of freedom are reduced to only one or two with the consequent loss of dexterity of the hand. The Kapandji opposition test (KOT) has been clinically used in pathological human hands for evaluating the thumb opposition and it has also been employed in some previous studies as reference for the design of the TKC in artificial hands, but without a clearly stated methodology. Based on this approaches, in this study we present a computational method to optimize the whole TKC (base placement, link lengths and joint orientation angles) of an artificial hand based on its performance in the KOT. The cost function defined for the optimization (MPE) is a weighted mean position error when trying to reproduce the KOT postures and can be used also as a metric to quantify thumb opposition in the hand. As a case study, the method was applied to the improvement of the TKC of an artificial hand developed by the authors and the MPE was reduced to near one third of that of the original design, increasing significantly the number of reachable positions in the KOT. The metric proposed based on the KOT can be used directly or in combination with other to improve the kinematic chain of artificial hands.
\end{abstract}

Keywords: Artificial Hand, Kinematic Chain, Optimization.

\section{Introduction}

The human hand is a marvelous tool optimized in an evolutionary process since our ancestors [1,2]. Thumb opposition is said to be one distinctive feature of the human hand. Interestingly, this dexterity can be obtained even with an important variability in the thumb anatomy among individuals [3]. The human thumb is composed of three bones (Fig. 1) [4]: the distal phalanx, the proximal phalanx and the first metacarpal bone, connected to the wrist. The interphalangeal joint (IP) is a hinge joint with one degree of freedom (DoF) whereas the metacarpo-phalangeal joint (MCP) is condylar and the carpo-metacarpal joint (CMC) is of saddle type, both with two DoFs. Therefore, the thumb's kinematic chain (TKC) can be considered as an open chain connected to the wrist with 5 DoFs, allowing a high range of positions and orientations of the 
thumb tip. It has been shown that the assumption of universal joints (two perpendicular and intersecting axes of rotation) for the CMC and MCP joints is not realistic and that a biomechanical model with five links [4], including two virtual links in these joints and considering non-orthogonal and non-intersecting axes in the joints is more realistic and represents better the anatomical evidences [3].

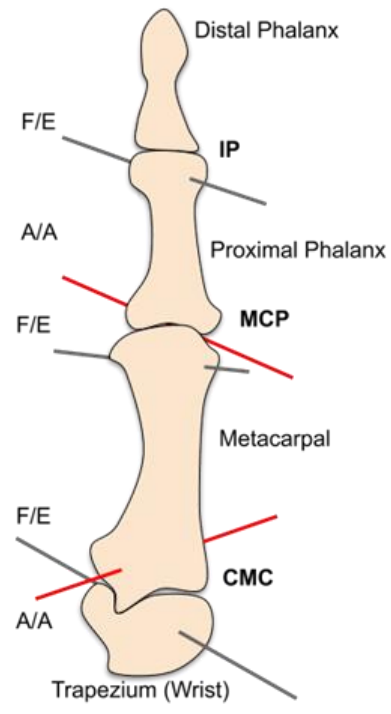

Fig. 1. Bones and joints of the human thumb [4].

The design of artificial hands, both prosthetic and robotic, is moving in last decades towards devices more anthropomorphic, to improve the functionality and the cosmetic appearance. Given the complexity of the TKC above explained, the designers of artificial hands need to introduce simplifications for this digit. These simplifications are mainly guided by the difficulty of obtaining adequate mechanical solutions for reproducing the geometry and mobility of the CMC and MCP joints, but also by the attempt to simplify the artificial hand control. Generally, the five DoFs of the human thumb are simplified in mechanical hands to achieve two basic motions: flexion/extension and circumduction. The circumduction rotation of the thumb is the movement requested to change the type of opposition of the thumb with respect to the long fingers, it allows to alternate between a lateral grasp and a power or precision grasp. In the human hand, the circumduction motion is achieved through a combination of 3 joints at the base of the thumb [5]. Belter et al. [6] reviewed the thumb design and position for different prosthetic hands. They highlighted the relevance of the relationship between the circumduction rotation axis of the thumb and the main axis of the wrist for functional grasps. In most of the prosthetic hands that Belter et al. analyzed, the thumb is actuated with a simple closing or opening (flexion/extension) and along the circumduction rotation axis, that is not always oriented parallel with the wrist rotation axis. They recommended to jointly approximate in a single DoF the 
thumb flexion and circumduction rotation for keeping complexity low. Ten Kate et al. [7] reviewed the kinematic specifications of 3D-printed hand prostheses and specified the range of motion for the thumb flexion and thumb circumduction of 58 devices. Three of the hands analyzed lack a thumb, $7 \%$ of the thumbs of the other hands did not perform flexion movement and $62 \%$ did not perform circumduction movement. Grebenstein et al. [8] analyzed anatomy, surgery and rehabilitation data for defining some guidelines to be used for the design of a robotic thumb for the DLR hand. They proposed a minimum of $3 \mathrm{DoF}$ to allow proper manipulation.

From the literature of both robotic and prosthetic fields, we can find thumbs with different mechanical configurations, changing the number of links and DoFs. Fig. 2 shows several representative examples.

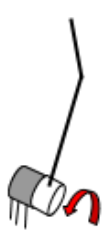

(a)

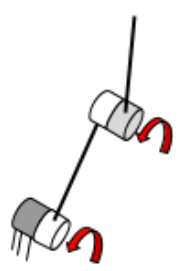

(b)

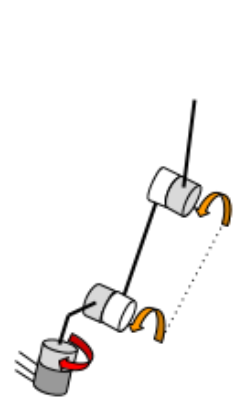

(c)

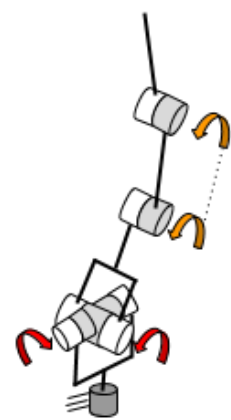

(d)

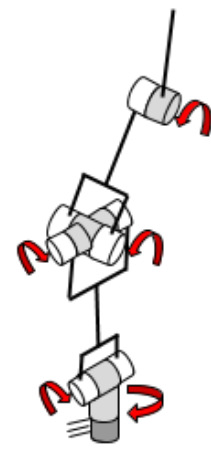

(e)

Fig. 2. Thumb's kinematic chain (TKC) for several artificial hands. a: SensorHand Speed [9], b: FRH-4 Hand [10], c: Bebionic hand [9], d: DLR/HIT Hand II [11], e: Shadow Dexterous Hand [12]. Red arrow: actuated by an independent actuator; yellow arrow: several DoFs actuated by the same actuator.

The thumb of the SensorHand Speed [9] (Fig. 2a) is a rigid bar with only 1 DoF. The thumb of the FRH-4 Hand of the mobile-assisting robot ARMAR [10] (Fig. 2b) has 2 DoFs actuated by two independent fluidic actuators that produce flexion motion of the MCP and IP joints, respectively. The thumb of the Bebionic hand [9] (Fig. 2c) has 3 DoFs, one actuator produce the flexion of the MCP and IP joints and the MCP circumduction has two selectable fixed positions, manually placed by the user of the prosthesis. The thumb of the DLR/HIT Hand II [11] (Fig. 2d) has 4 DoFs and 3 actuators, one for the CMC flexion, other for the MCP and IP flexion and other for the CMC abduction. The thumb of the Shadow Dexterous Hand [12] (Fig. 2e) has 5 independently actuated DoFs, as the human hand, but the MCP and CMC are universal joints.

It could be interesting to have objective methods to evaluate the impact of simplifications made in the thumb of artificial hands in the loss of ability to grasp in real life applications. These objective methods could help designers to obtain hand designs with improved grasping abilities. The Kapandji opposition test (KOT) [13], also 
called total opposition test, can be of interest for this goal. The KOT was proposed as a simple method for assessing the opposition of the thumb in pathological hands and is used in current clinical practice. It involves touching different areas of the four long fingers with the tip of the thumb (Fig. 3). The score obtained in the test ranges from 1 to 10 depending on the last reached area, being the test performed in the order indicated in Table 1.

Table 1. Scores according to the Kapandji opposition test (KOT)

\begin{tabular}{cll}
\hline Score & Finger & Area of contact \\
\hline 1 & Index & Lateral side of the second phalanx \\
2 & Index & Lateral side of the third phalanx \\
3 & Index & Tip \\
4 & Middle & Tip \\
5 & Ring & Tip \\
6 & Little & Tip \\
7 & Little & DIP crease \\
8 & Little & PIP crease \\
9 & Little & Proximal crease \\
10 & - & Distal volar crease of the hand \\
\hline
\end{tabular}

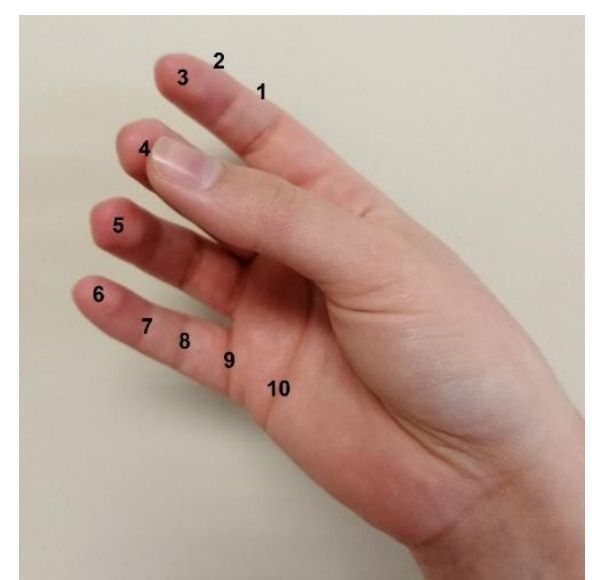

Fig. 3. Areas to touch with the thumb tip in the Kapandji opposition test (KOT)

Grebenstein et al. [8] considered that the KOT includes motion of the fingers and the thumb sufficient to evaluate the manipulation abilities. Other authors used the KOT to evaluate the functionality and anthropomorphism of artificial hands. Shin et al. [14] used the KOT to analytically analyze a new dexterous robot hand for delicate object grasping. Chalon et al. [15] used the KOT to optimize the thumb of the Awiwi Hand obtaining the maximum score at KOT. Roa et al. [16] explored the relationship between kinematic design and manipulation performance of robotic hands, to analyze it 
they performed the KOT on seven thumb placements of a modular robotic hand. Deimel et al. [17] also assessed the dexterity of the opposable thumb of a soft robotic hand using the KOT. Cerruti et al. [18] used the KOT to validate the thumb base placement of a kinematic model of an anthropomorphic robotic hand used for gesturing and grasping.

Some of the above mentioned studies that used the KOT made some adaptation of the test. For example, in some studies the authors did not consider the positions 1 and 2 corresponding to contact of the thumb with the lateral side of the index $[16,17]$ or removed some positions in the little finger [17]. Contrary, in some cases additional positions in the middle and ring fingers are included [16,17]. In most of these studies the KOT is only used to evaluate different hand designs. In some of them the authors used the KOT to optimize the thumb base placement $[15,16,18]$. In [15] the optimization of the thumb included also as parameters the orientation of the joints, but the details about the cost function are not provided. To our knowledge, no previous study made an optimization of the TKC including simultaneously base placement, link lengths and all the joint orientation angles.

Following these approaches, in this study the objective was to define a computational method to optimize the whole TKC (base placement, link lengths and joint orientation angles) of an artificial hand based on its performance in the KOT. This method could be useful to improve the design of prosthetic and robotic hands regarding thumb opposition, leading to a better object grasping and manipulation. The application of the method involves defining an index, used in the cost function for optimization, which provides a metric for rating thumb opposition in artificial hands. To test the method we applied it to a 3D-printed prosthetic hand developed by the authors: the IMMA hand [19].

\section{Materials and Methods}

\subsection{IMMA Hand}

The IMMA hand [19] is a low-cost tendon-driven anthropomorphic prosthetic hand designed by the authors. It has five fingers with three phalanges per finger and $6 \mathrm{DoFs}$ in total: independent flexion/extension in each of the four long fingers, and two independent DoFs for the thumb. The MCP and IP joints of the thumb are actuated both with the same tendon for flexion and the $\mathrm{CMC}$ joint is actuated by a separate tendon for circumduction. Fig. 4 shows the TKC of the IMMA hand. Fig. 5 shows the achievable target areas of the KOT by the right IMMA hand prototype. As is shown, its score is 4 , because the positions 5 to 10 (see Table 1) cannot be reached. 


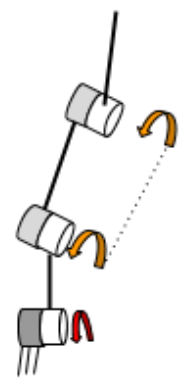

Fig. 4. Thumb's kinematic chain (TKC) of the IMMA hand.

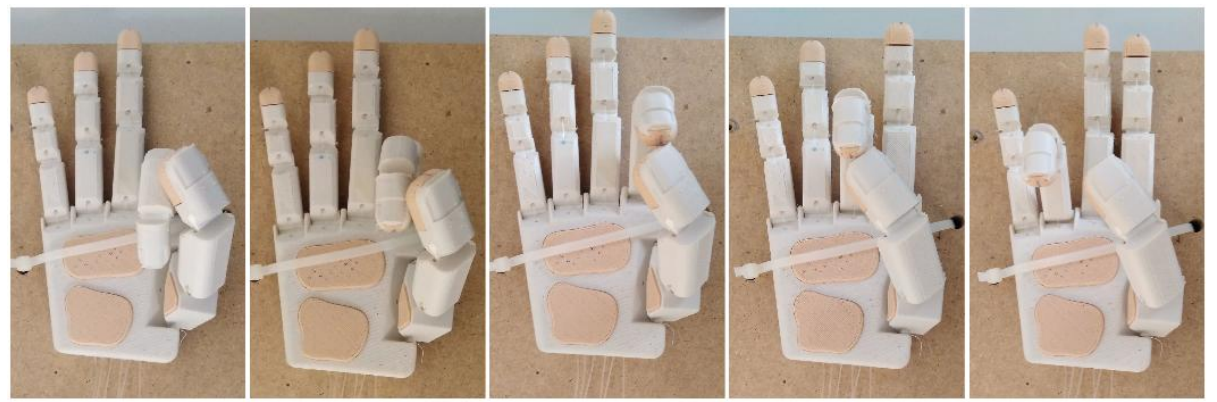

Fig. 5. IMMA hand performing the Kapandji opposition test (KOT) in positions 1 (left) to 5 (right).

\subsection{Computational Model}

Hand Model. The model of the hand used in this study has a maximum of four straight links for each digit and a maximum of five digits. To define the kinematic chain of the hand, a local coordinate system (LCS) for each link has to be defined. In this study the LCS of each link is located in the middle of the joint with the proximal link. The LCSs were defined under the following criteria: Z-axis coincident with the flexion/extension axis of the joint, and oriented so that the flexion motion corresponds to a positive rotation around $\mathrm{Z}, \mathrm{X}$-axis aligned in palmar-dorsal direction pointing dorsally, thus indicating the abduction/adduction axis in the joint and Y-axis defining a right-handed coordinate system with the previous ones, resulting in a distal direction, in other words, pointing in the direction towards the tip of the fingers. This convention for the orientation of the axes is similar to that proposed by the ISB [20], with the difference that the $\mathrm{X}$ and $\mathrm{Y}$ axes have opposite positive directions. With this selection the position of each LCS relative to the proximal one in the kinematic chain presents positive values in the translation along the $\mathrm{Y}$ axis.

Therefore, the kinematic chain of the hand is defined in the reference position with the three displacements and rotations of each LCS with respect to the immediate proximal in the chain. The wrist is taken as the fixed LCS for all the digits. Specifically, 
the LCS of link $j$ (1: metacarpus, 2: proximal phalanx, 3: intermediate phalanx, 4 : distal phalanx) of the digit $i$ (1: thumb, 2: index, 3: middle, 4: ring, 5: little) is defined with the Equation 1.

$$
v_{i, j}=\left[\begin{array}{llllll}
x_{i, j} & y_{i, j} & z_{i, j} & \theta_{x_{i, j}} & \theta_{y_{i, j}} & \theta_{z_{i, j}}
\end{array}\right], \quad i=1,2 \ldots, 5, \quad j=1,2 \ldots, 5
$$

where the first three elements of the vector correspond to the translation vector of $\mathrm{LCS}_{\mathrm{i}, \mathrm{j}}$ with respect to $\mathrm{LCS}_{\mathrm{i}, \mathrm{j}-1}$ and the last three to the Euler angles with sequence of rotations XZY to orient $\mathrm{LCS}_{\mathrm{i}, \mathrm{j}-1}$ as $\mathrm{LCS}_{\mathrm{i}, \mathrm{j}}$. At each finger, a last LCS $(\mathrm{j}=5)$ is added, positioned at its end (fingertip), with its $\mathrm{Y}$ axis in the proximal-distal direction and its $\mathrm{X}$ axis in the palmar-dorsal direction.

For each digit a maximum of six DoFs can be included in the hand model, two in $\mathrm{CMC}$ and MCP joints and one in the IP joints. Universal joints are considered in those with two DoFs. The hand position can be obtained straightforward by direct kinematics using the Equation 1 and the rotation angles in the joints.

Cost Function. To optimize the TKC we defined a cost function based on the KOT postures. We defined the position error $\varepsilon_{i}$ for each posture $i$ of the KOT as the minimum possible distance between the thumb tip and the corresponding target point of the test while the hand is moved within its workspace (Equation 2).

$$
\varepsilon_{i}=\min \left(\operatorname{dist}\left(p_{i}, p_{t}\right)\right)
$$

where $p_{i}$ is the target point, $p_{t}$ the thumb tip point, dist is a function defining the distance between two points and $\min$ is a function obtaining the minimum possible value of dist when moving the hand within its workspace.

Given a hand geometry and the range of motion of their joints, the calculation of the $\varepsilon_{i}$ involves an optimization. The variables for this optimization are the joint rotation angles of the hand. If these angles are coupled with a linkage, the number of variables for the optimization can be reduced, because the coupled rotation angles can be obtained from the coupling equations.

The final cost function for the optimization of the TKC was defined with Equation 3 as a relative mean position error (MPE) for the different positions of the KOT.

$$
M P E=\sum_{i} \varepsilon_{i} w_{i}
$$

where $w_{i}$ is a weighting coefficient for the posture $i$ of the KOT. Dividing the lengths of the kinematic chain by the hand length is convenient for having a nondimensional index and making the evaluation independent of the hand size.

Optimization algorithm. For optimizing the TKC based on the KOT, the MPE above defined (Equation 3) has to be minimized, being the optimization variables the parameters defining the TKC: base placement, joint angles orientation and links length. Depending on the designer interest, it is also possible to limit the variables to only some of those defining the TKC. As the calculation of the minimum MPE requires the previous computation of the position errors $\varepsilon_{i}$ (Equation 2), the computational model 
involves two nested optimizations. Matlab was used in this study for the computation. The built-in Genetic Algorithm (' $g a$ ') was used for the optimization of the TKC whereas 'fmincon' function for non-linear optimization was used for the nested optimization corresponding to each position of the KOT. Table 2 shows the pseudocode used in the computation model.

Table 2. Pseudocode for optimizing the TKC.

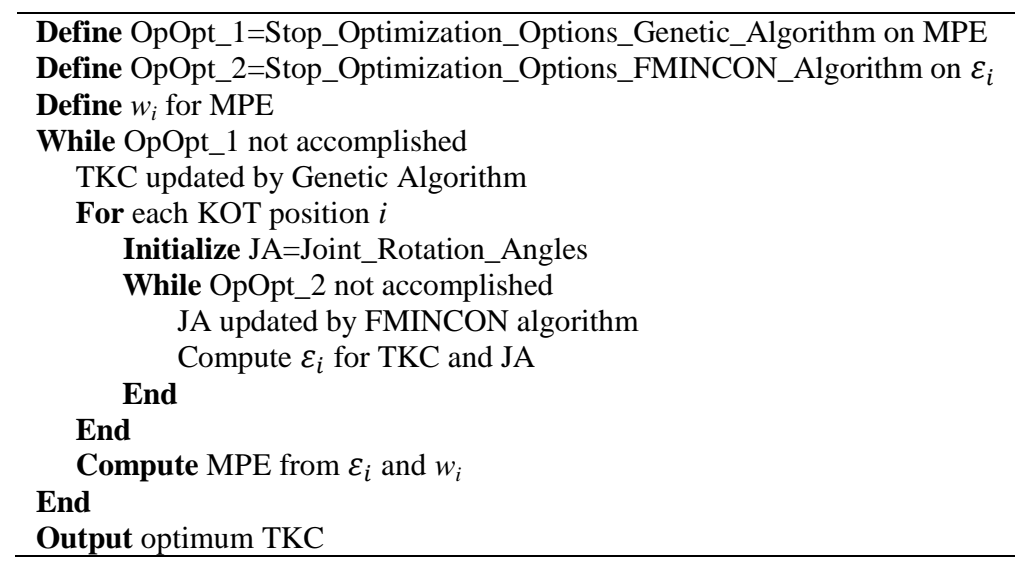

\subsection{Case study: Optimization of the TKC of the IMMA hand}

A simplified model of the IMMA hand [19] was created in Matlab (Fig. 6). Table 3 shows the components of the translation-rotation vectors that define the kinematic chain of the hand (vectors $v_{i, j}$, Equation 1), where $x, y, z$ are non-dimensional values related to the hand length (distance from the wrist to the end of the middle finger) and $\theta_{x}, \theta_{y}, \theta_{z}$ angles are the Euler rotations around the $\mathrm{X}, \mathrm{Y}, \mathrm{Z}$ axes, respectively, with rotation order XZY, expressed in radians. The joints range of motion (ROM) were defined based on the hand prototype (Fig. 5) and are shown in Table 4. For the abduction/adduction movement in the MCP joints we included a small ROM accounting for the flexibility of the joints in the prototype.

Table 3. Data for the kinematic chain of the IMMA hand according to Equation 1 (lengths are non-dimensional values related to the hand length and Euler angles are in radians).

\begin{tabular}{lcccccc}
\hline Links & $v_{i, j}$ & Thumb & Index & Middle & Ring & Little \\
\hline Metacarpal & $x$ & 0 & 0 & 0 & 0 & 0 \\
& $y$ & 0.2169 & 0 & 0 & 0 & 0 \\
& $z$ & 0.1577 & 0 & 0 & 0 & 0 \\
& $\theta x$ & 1.5708 & 0 & 0 & 0 & 0 \\
& $\theta y$ & 0 & 0 & 0 & 0 & 0 \\
& $\theta z$ & 0 & 0 & 0 & 0 & 0 \\
\hline Proximal phalanx & $x$ & 0 & 0 & 0 & 0 & 0 \\
& $y$ & 0.1320 & 0.4588 & 0.4370 & 0.4192 & 0.3874
\end{tabular}




\begin{tabular}{lcccccc} 
& $z$ & 0 & 0.1188 & 0.0016 & -0.1054 & -0.2004 \\
& $\theta x$ & 0 & 0.0873 & 0 & -0.1250 & -0.2618 \\
& $\theta y$ & -0.7854 & 0 & 0 & 0 & 0 \\
& $\theta z$ & 0 & 0 & 0 & 0 & 0 \\
\hline Intermediate phalanx & $x$ & 0 & 0 & 0 & 0 & 0 \\
& $y$ & 0.2158 & 0.2456 & 0.2725 & 0.2291 & 0.1968 \\
& $z$ & 0 & 0 & 0 & 0 & 0 \\
& $\theta x$ & 0 & 0 & 0 & 0 & 0 \\
& $\theta y$ & 0 & 0 & 0 & 0 & 0 \\
\hline Distal phalanx & $\theta z$ & 0 & 0 & 0 & 0 & 0 \\
& $x$ & 0 & 0 & 0 & 0 & 0 \\
& $y$ & 0.1659 & 0.1399 & 0.1623 & 0.1509 & 0.0590 \\
& $z$ & 0 & 0 & 0 & 0 & 0 \\
& $\theta x$ & 0 & 0 & 0 & 0 & 0 \\
\hline Fingertip & $\theta y$ & 0 & 0 & 0 & 0 & 0 \\
& $\theta z$ & 0 & 0 & 0 & 0 & 0 \\
\hline & $x$ & 0 & 0 & 0 & 0 & 0 \\
& $y$ & 0 & 0.1243 & 0.1324 & 0.1324 & 0.1135 \\
& $z$ & 0 & 0 & 0 & 0 & 0 \\
& $\theta x$ & 0 & 0 & 0 & 0 & 0 \\
& $\theta y$ & 0 & 0 & 0 & 0 & 0 \\
& $\theta z$ & 0 & 0 & 0 & 0 & 0 \\
\hline
\end{tabular}

Table 4. Joints range of motion (minimum angle/maximum angle) in degrees for the IMMA hand (add/abd: adduction/abduction, ext/flex: extension/flexion).

\begin{tabular}{lccccc}
\hline Joint & Thumb & Index & Middle & Ring & Little \\
\hline CMC add/abd & $0 / 0$ & $0 / 0$ & $0 / 0$ & $0 / 0$ & $0 / 0$ \\
CMC ext/flex & $-10 / 70$ & $0 / 0$ & $0 / 0$ & $0 / 0$ & $0 / 0$ \\
MCP add/abd & $-1 / 1$ & $-1 / 1$ & $-1 / 1$ & $-1 / 1$ & $-1 / 1$ \\
MCP ext/flex & $-11 / 55$ & $-20 / 85$ & $-17 / 86.3$ & $-15 / 70$ & $-20 / 65$ \\
PIP ext/flex & $-13 / 55$ & $-20 / 60$ & $-17 / 75$ & $-20 / 75$ & $-20 / 65$ \\
DIP ext/flex & $0 / 0$ & $-15 / 50$ & $-20 / 75$ & $-20 / 70$ & $-20 / 75$ \\
\hline
\end{tabular}




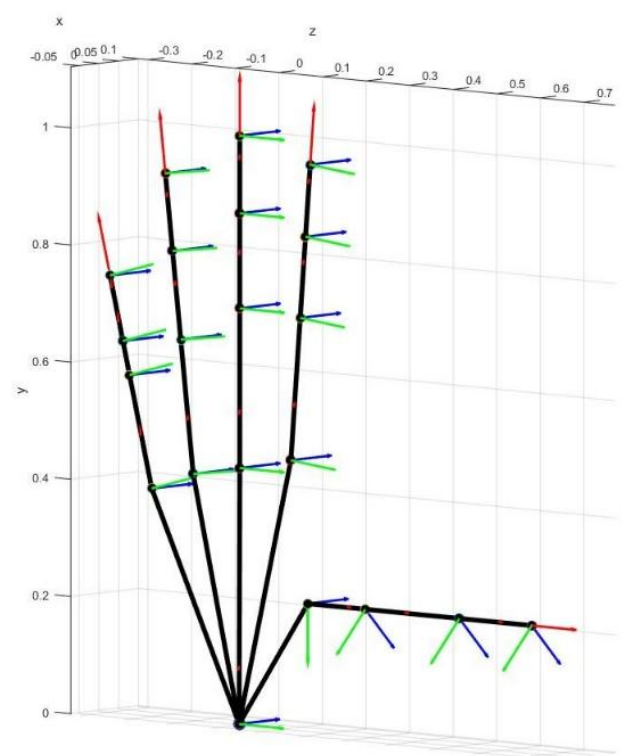

Fig. 6. Simplified model of the right IMMA hand. Colored arrows in the joints indicate local coordinate systems (LCSs): green for Z-axis, red for $\mathrm{Y}$-axis, blue for $\mathrm{X}$-axis.

For the optimization of the TKC in this case study the position 10 of the KOT (see Fig. 3) was not considered because it was difficult to locate in a simplified model of the hand. The positions 1 and 2 where considered in the more proximal point of the corresponding index phalanx. In Equation 3 positions considered were weighted equally, so we used $w_{i}=1 / 9$ for $i=1$ to 9 and $w_{10}=0$. Moreover, in this case the joint rotation angles of the hand were considered independent, without taking into account the coupling equations resulting from the actuation of several joints with a same tendon. The variables for the optimization were the orientation of the CMC and MCP joints, the thumb's links length and the position of the CMC joint. The feasible range of these variables, upper and lower bounds for the optimization, is shown in Table 5. The default optimization options were considered for the genetic algorithm of Matlab (' $g a$ ') except 'FitnessLimit' and 'FunctionTolerance' both set to 0.001 and 'PopulationSize' set to 50. For the non-linear optimization with Matlab built-in function ('fmincon') the default optimization options were also considered except 'MaxFunctionEvaluations' set to 10000 and 'StepTolerance' set to 0.0001 .

Table 5. Lower and upper bounds (Min/Max) of the optimization variables in this case study (lengths are non-dimensional values related to the hand length and Euler angles are in radians).

\begin{tabular}{lccl}
\hline$v_{i, j}$ component & Min & Max & Anatomical meaning \\
\hline$x_{1,1}$ & -0.3 & 0 & CMC position \\
$y_{l, 1}$ & 0.1 & 0.3 & \\
$z_{l, 1}$ & 0.1 & 0.3 & \\
\hline
\end{tabular}




\begin{tabular}{lccl}
\hline$\theta x_{1,1}$ & $\pi / 2$ & $\pi$ & CMC orientation \\
\hline$y_{1,2}$ & 0.1 & 0.3 & Metacarpal length \\
\hline$\theta y_{1,2}$ & $-\pi / 2$ & $\pi / 2$ & MCP orientation \\
\hline$y_{1,3}$ & 0.1 & 0.3 & Proximal phalanx length \\
\hline$y_{1,4}$ & 0.1 & 0.3 & Distal phalanx length \\
\hline
\end{tabular}

\section{$3 \quad$ Results}

Fig. 7 shows the position error $\left(\varepsilon_{i}\right.$, Eq. 2$)$ for the the initial IMMA hand and for the IMMA hand after optimizing the TKC following the method indicated in Table 2. According to the simplified model the position 1 of the KOT is not reachable by the original hand nor by the improved one. Without considering this position, the score in the KOT improved from 3 in the original hand to 5 in the model with optimized TKC. The position error for positions 6 to 8 improved significantly. The MPE in the optimized model was reduced to about one third with respect to the original model $(0.121$ to 0.035$)$.

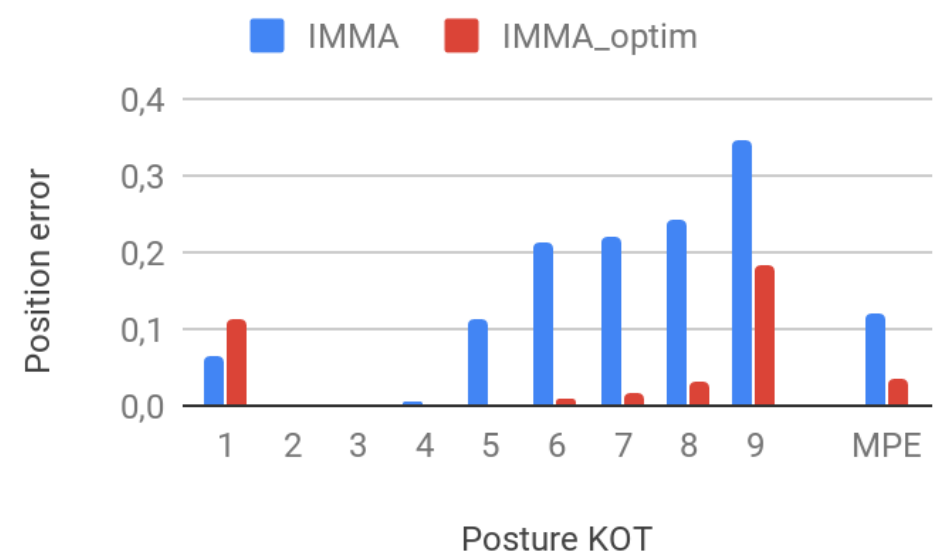

Fig. 7. Position error ( $\varepsilon_{i}$, Eq. 2$)$ for each posture of KOT and mean position error (MPE).

The kinematic chain of the improved hand can be seen in Fig. 8 and the Table 6 shows the comparison of the initial and optimized values of the parameters for the TKC. From the comparison of the thumb in Fig. 6 and Fig. 8 it can be observed that the base placement of the thumb, i.e. the $\mathrm{CMC}$ joint, is closer to the center of the palm in the optimized design, the orientation of the MCP joint is slightly varied and the phalanges length has changed, being the distal phalanx longer in the optimized design. 


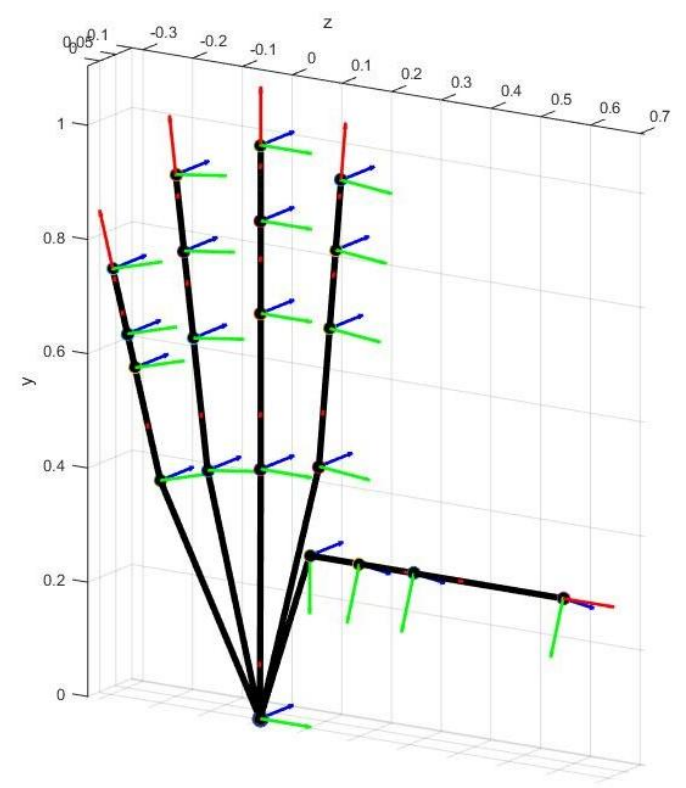

Fig. 8. Kinematic chain of the right IMMA hand with optimized TKC.

Table 6. Initial and optimized parameters of the TKC for the IMMA hand (lengths are nondimensional values related to the hand length and Euler angles are in radians).

\begin{tabular}{ccc}
\hline$v_{i, j}$ component & Initial & Optimized \\
\hline $\mathrm{x} 1,1$ & 0.000 & 0.000 \\
$\mathrm{y} 1,1$ & 0.2169 & 0.3000 \\
$\mathrm{z} 1,1$ & 0.1577 & 0.1002 \\
$\theta \mathrm{x} 1,1$ & 1.5708 & 1.5714 \\
$\mathrm{y} 1,2$ & 0.1320 & 0.1000 \\
$\theta \mathrm{y} 1,2$ & -0.7854 & -0.3855 \\
$\mathrm{y} 1,3$ & 0.2158 & 0.1094 \\
$\mathrm{y} 1,4$ & 0.1659 & 0.3000 \\
\hline
\end{tabular}

Fifty-nine generations were necessary in the genetic algorithm for the optimization of the TKC and the execution took about 10 hours in an Intel Core i7 $2.6 \mathrm{GHz}$ processor. Fig. 9 shows the evolution of the mean and best fitness values, corresponding to MPE, for the different generations of the genetic algorithm. The 50 individuals of the last generation of the genetic algorithm were all very similar among them, representing quite similar TKCs. 


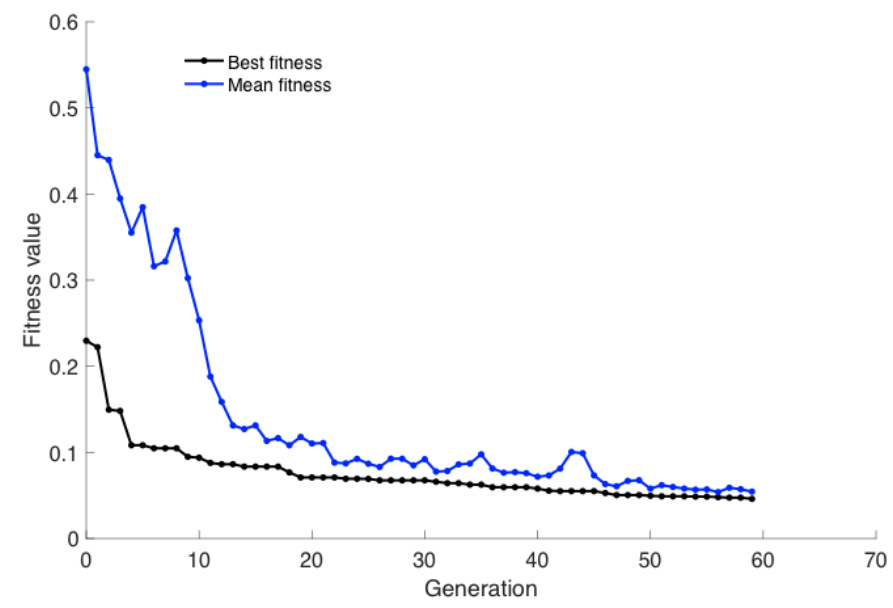

Fig. 9. Fitness value (MPE) evolution among generations of the genetic algorithm for optimizing the thumb's kinematic chain (TKC).

\section{Discussion}

We have presented a new method to optimize the TKC of an artificial hand based on their performance in the KOT. Despite the KOT has been taken into account in previous studies for the design of artificial hands $[15,16,18]$ none of these previous studies presented a clear computational method able to do it following a formal optimization procedure and including all the parameters defining the TKC. The methodology presented here, using a double nested optimization method (Table 2) allows considering all the KOT postures for the optimization or only some of them. We have defined an index quantifying the mean weighted position error (MPE) based on the position error for each KOT posture, which can be useful as a method to compare the opposition performance of an artificial hand. The weight associated to each posture in this index can easily be tuned by the designer depending on their design specifications. Moreover, the optimization procedure allows including as variables any of the parameters defining the TKC or even other parameters of the kinematic chain of the hand.

In this study the methodology was applied, as a case study, to the optimization of the TKC of the IMMA hand, a 3D-printed cable driven hand developed by the authors. The MPE in the optimized design was reduced to less than one third of that of the original design, showing the effectiveness of the computational method. Due to the simplification of the hand model, whose segments are considered as straight lines, the computational model is only an approximation to the real prototype and some differences can be observed in the KOT score obtained with the real prototype and with the model. As Fig. 5 shows, the original prototype of the IMMA hand, can achieve the positions 1 to 4 of the KOT. Nevertheless, Fig. 7 shows a non-null position error in the model for positions 1 and 4 . This could be attributed to the fact that the width and thickness of the phalanges were neglected in the simplified model and also to the fact that positions 1 and 2 where considered in the more proximal point of 
the corresponding index phalanx. Taking this into account, the positions 2 to 8 in the improved model can be considered as reachable in practical terms, whereas only the positions 2 to 4 are clearly reachable in the initial hand. Regarding the position 1, it could also be reachable depending on the position taken for the target point in the middle phalange of the index finger (Fig. 10).
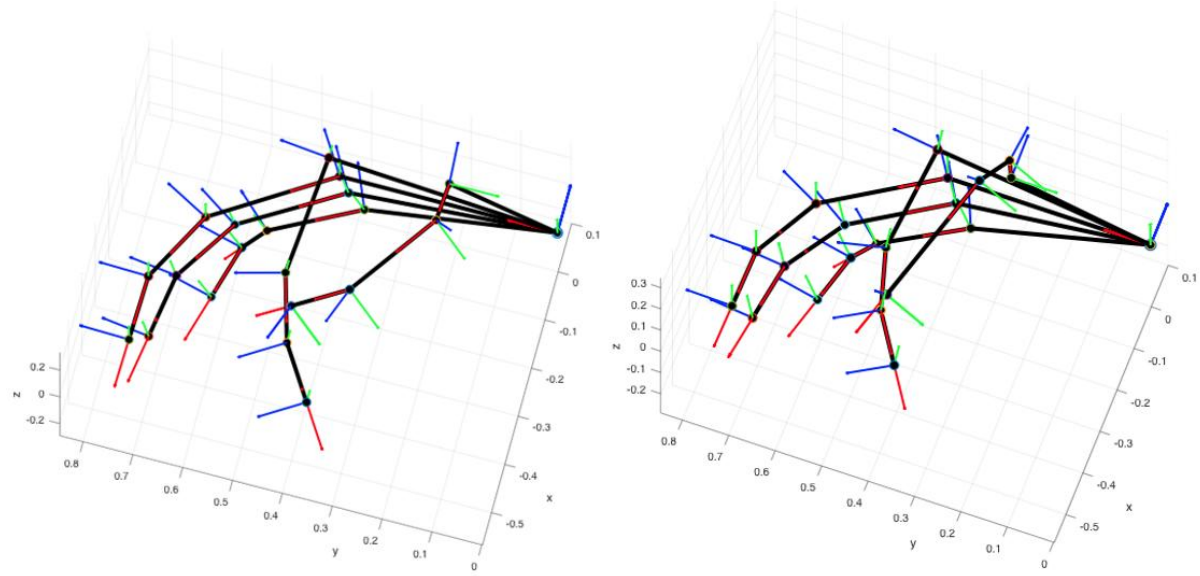

Fig. 10. Posture 1 of the Kapandji opposition test (KOT). Left: original IMMA hand. Right: optimized IMMA hand.

Some of the parameters of the optimized model (Table 6) are in the upper or lower bounds selected in the optimization (Table 5), which could indicate that widening the allowable range for the parameters could produce TKC designs able to reduce even more the MPE. This has not been analyzed in the present study but is a possible future work.

The optimized design obtained in this study has a thumb base location more distal, favoring the score in the KOT, but probably making more difficult grasping big objects. The total length of the optimized TKC is similar to that of the original design, but the proportion of the segments changed, with a longer distal phalanx and shorter metacarpal and proximal phalanges as compared to the original model. One possible reason for this is that this configuration helps to reduce the position error in the last postures of the KOT (7 to 9). We analyzed the changes in the results when the KOT positions included in the MPE are restricted to positions 1 to 6 , reducing the effect of little finger opposition in the MPE. Fig. 11 shows a graphical comparison of the TKC parameters of the original design and the optimized designs including positions 1 to 9 or 1 to 6 in the MPE, as well as the upper and lower bounds considered for the optimization. The results indicate that the optimized TKCs including positions 1 to 9 or 1 to 6 are very similar. 


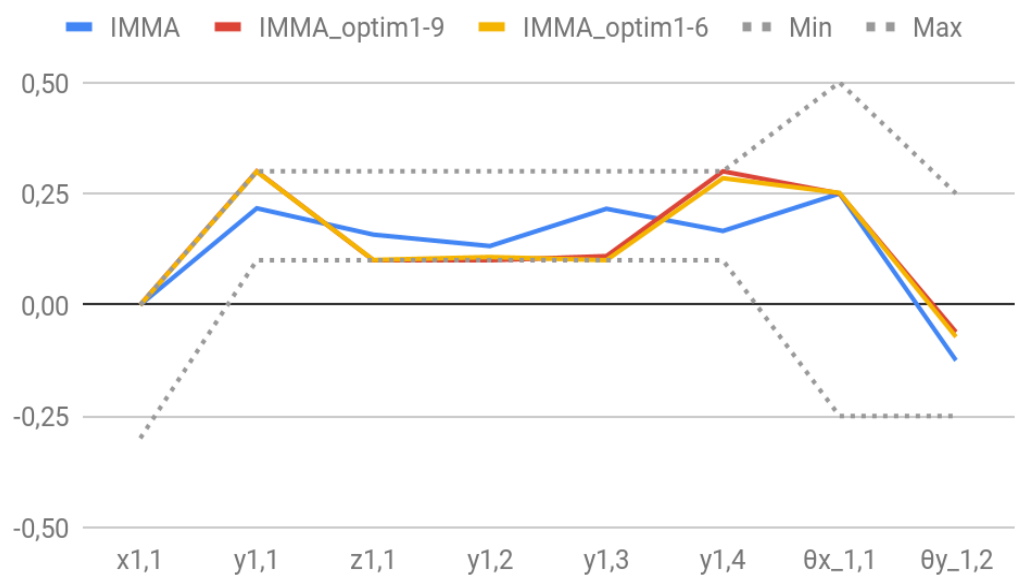

Fig. 11. Thumb's kinematic chain (TKC) parameters (angles $\theta_{x_{-} l, 1}$ and $\theta_{y_{-} l, 2}$ divided by $2 \pi$ ) for the original design of the IMMA hand and for the optimized versions obtained including positions 1-9 or 1-6 in the MPE. Upper (Max) and lower (Min) bounds for the optimization are shown with dotted lines.

Additional simulations in Matlab for the original IMMA hand and the optimized design suggest that the reduction of MPE in KOT does not guarantee a better design according to other criteria when comparing with the human hand. We compared both designs with three different anthropomorphic indexes of the kinematic chain of the whole hand [21] and the performance was similar, with differences lower to $3 \%$, for two of them: one based on the comparison of the kinematic chain with that of the human hand; a second based on grasping postures for some primitive objects (sphere, cylinder and prism). However, the optimized design had a worse performance, $25 \%$ lower, in the index defined considering the intersection of the workspaces of the artificial hand and the human hand. This result is aligned with the observation of Roa et al. [16] about the difficulties to find direct correlations between the Kapandji test score and the size of the functional workspace. This aspect should be better investigated in the next future. The performance of the optimized design of the IMMA hand with respect to the original design should also be compared in the next future with physical prototypes, using grasping benchmarks.

The index based on the MPE defined in this study is complementary to other anthropomorphism indexes developed by the authors and existing in the literature. Probably an adequate combination of these indexes can help to improve the hand's kinematic chain and should be investigated, as well as the extension of the optimization to other parameters of the kinematic chain of the hand, not restricted to the thumb. 


\section{Conclusion}

A straightforward methodology to analytically optimize the kinematic chain of the thumb of an artificial hand based on the performance in the KOT has been presented. The cost function defined for the optimization (MPE) is a weighted mean position error when trying to reproduce the KOT postures and can be used also as a metric to quantify thumb opposition in the hand. The application of the method to the IMMA hand thumb allowed defining a new TKC reducing the MPE to near one third of that of the original design and increasing significantly the number of reachable positions in the KOT. However, additional simulations showed that the optimized design could have a worse outcome according to other index considering the intersection between the workspace of the artificial hand and that of the human hand. Benchmarking grasping test on physical prototypes could give additional insights about the usefulness of the proposed methodology. The metric proposed based on the KOT can be used directly or in combination with other to improve the kinematic chain of artificial hands.

Acknowledgments. This work was supported by the Spanish Ministry of Economy and Competitiveness and ESF [grant number BES-2015-076005]; Spanish Ministry of Economy and Competitiveness, AEI and ERDF [grant numbers DPI2014-60635-R, DPI2017-89910-R]; and Universitat Jaume I [grant number UJI-B2017-70].

\section{References}

1. T. Feix, T. L. Kivell, E. Pouydebat, and A. M. Dollar, 'Estimating thumb-index finger precision grip and manipulation potential in extant and fossil primates', J. R. Soc. Interface, vol. 12, no. 106, pp. 20150176-20150176, Apr. 2015.

2. A. Bardo, L. Vigouroux, T. L. Kivell, and E. Pouydebat, 'The impact of hand proportions on tool grip abilities in humans, great apes and fossil hominins: A biomechanical analysis using musculoskeletal simulation', J. Hum. Evol., vol. 125, pp. 106-121, Dec. 2018.

3. V. J. Santos and F. J. Valero-cuevas, 'to Multimodal Distributions of Denavit-Hartenberg Parameters for the Human Thumb', IEEE Trans. Biomed. Eng., vol. 53, no. 2, pp. 155$163,2006$.

4. D. J. Giurintano, A. M. Hollister, W. L. Buford, D. E. Thompson, and L. M. Myers, 'A virtual five-link model of the thumb', Med. Eng. Phys., vol. 17, no. 4, pp. 297-303, 1995.

5. J. H. Coert, G. A. H. van Dijke, S. E. R. Hovius, C. J. Snijders, and M. F. Meek, 'Quantifying thumb rotation during circumduction utilizing a video technique', J. Orthop. Res., vol. 21, no. 6, pp. 1151-1155, Nov. 2003.

6. J. T. Belter, J. L. Segil, A. M. Dollar, and R. F. Weir, 'Mechanical design and performance specifications of anthropomorphic prosthetic hands: A review', J. Rehabil. Res. Dev., vol. 50, no. 5, p. 599, 2013.

7. J. ten Kate, G. Smit, and P. Breedveld, '3D-printed upper limb prostheses: a review', Disabil. Rehabil. Assist. Technol., vol. 12, no. 3, pp. 300-314, Apr. 2017.

8. M. Grebenstein, M. Chalon, G. Hirzinger, and R. Siegwart, 'A Method for Hand Kinematics Designers 7 Billion Perfect Hands', 1st Int. Conf. Appl. Bionics Biomech., 2010. 
9. Ottobock, 'Ottobock myoelectric prosthetics', 2017. [Online]. Available: https://www.ottobockus.com/prosthetics/upper-limb-prosthetics/solutionoverview/myoelectric-prosthetics/. [Accessed: 01-Mar-2019].

10. I. Gaiser et al., 'A new anthropomorphic robotic hand', in Humanoids 2008 - 8th IEEERAS International Conference on Humanoid Robots, 2008, pp. 418-422.

11. H. Liu et al., 'Multisensory five-finger dexterous hand: The DLR/HIT Hand II', in 2008 IEEE/RSJ International Conference on Intelligent Robots and Systems, 2008, pp. 36923697.

12. Shadow Robot Company, 'Shadow Dexterous Hand', 2019. [Online]. Available: https://www.shadowrobot.com/products/dexterous-hand/. [Accessed: 01-Mar-2019].

13. A. Kapandji, 'Clinical opposition and reposition test of the thumb [COTATION CLINIQUE DE L'OPPOSITION ET DE LA CONTRE-OPPOSITION DU POUCE]', Ann. Chir. la Main, vol. 5, no. 1, pp. 67-73, 1986.

14. S. Shin, D. Choi, M. Choi, H. Moon, H. R. Choi, and J. C. Koo, 'Development of dexterous robot hand for delicate object grasping', in 2012 9th International Conference on Ubiquitous Robots and Ambient Intelligence (URAI), 2012, pp. 462-463.

15. M. Chalon, A. Dietrich, and M. Grebenstein, 'The Thumb of the Anthropomorphic Awiwi Hand: From Concept to Evaluation', Int. J. Humanoid Robot., vol. 11, no. 3, p. 1450019, Sep. 2014.

16. M. A. Roa et al., 'Towards a functional evaluation of manipulation performance in dexterous robotic hand design', in 2014 IEEE International Conference on Robotics and Automation (ICRA), 2014, pp. 6800-6807.

17. R. Deimel and O. Brock, 'A novel type of compliant and underactuated robotic hand for dexterous grasping', Int. J. Rob. Res., vol. 35, no. 1-3, pp. 161-185, Jan. 2016.

18. G. Cerruti, D. Chablat, D. Gouaillier, and S. Sakka, 'Design method for an anthropomorphic hand able to gesture and grasp', in 2015 IEEE International Conference on Robotics and Automation (ICRA), 2015, vol. 2015-June, no. June, pp. 3660-3667.

19. I. Llop-Harillo and A. Pérez-González, 'System for the experimental evaluation of anthropomorphic hands. Application to a new 3D-printed prosthetic hand prototype', Int. Biomech., vol. 4, no. 2, pp. 50-59, 2017.

20. G. Wu and P. R. Cavanagh, 'ISB recommendations for standardization in the reporting of kinematic data', J. Biomech., vol. 28, no. 10, pp. 1257-1261, 1995.

21. I. Llop-Harillo, A. Pérez-González, and F. J. Andrés de la Esperanza, 'Comparación de la cadena cinemática de manos antropomorfas artificiales con la de la mano humana', in Anales de Ingeniería Mecánica. Revista de la Asociación Española de Ingeniería Mecánica. Actas del XXII CONGRESO NACIONAL DE INGENIERÍA MECÁNICA., 2018, pp. 49-68. 